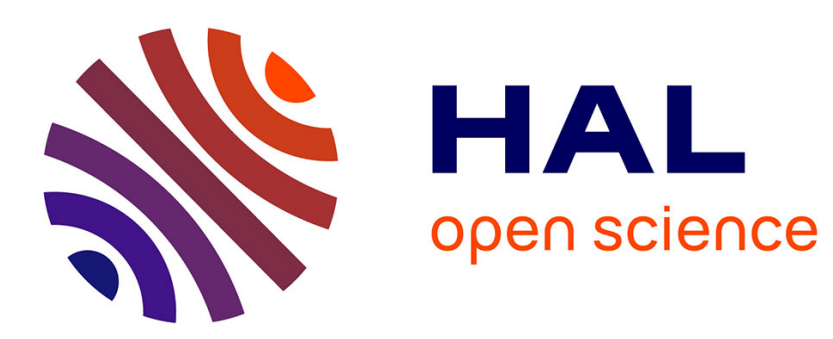

\title{
Guest Editorial - Special Section on In-vehicle Embedded Systems
}

Françoise Simonot-Lion

\section{To cite this version:}

Françoise Simonot-Lion. Guest Editorial - Special Section on In-vehicle Embedded Systems. IEEE Transactions on Industrial Informatics, 2009, Special Section on Communication in Automation, 5 (4), pp.372-374. 10.1109/TII.2009.2031747 . inria-00433072

\section{HAL Id: inria-00433072 https://hal.inria.fr/inria-00433072}

Submitted on 18 Nov 2009

HAL is a multi-disciplinary open access archive for the deposit and dissemination of scientific research documents, whether they are published or not. The documents may come from teaching and research institutions in France or abroad, or from public or private research centers.
L'archive ouverte pluridisciplinaire HAL, est destinée au dépôt et à la diffusion de documents scientifiques de niveau recherche, publiés ou non, émanant des établissements d'enseignement et de recherche français ou étrangers, des laboratoires publics ou privés. 


\section{Guest Editorial Special Section on In-vehicle Embedded Systems}

$\mathrm{O}$ ver the past two decades, there has been a significant increase in the number of computer based functions embedded in vehicles. While automobile production is likely to increase slowly in the coming years, the part of embedded electronics and more precisely embedded software is growing. In 2006, the electronic embedded system represented at least $25 \%$ of the total cost of a car and more than $35 \%$ for a highend model [1]. This trend has been driven by economical as well as technological factors. On the one hand, several local area networks were specifically developed for the automotive industry such as LIN [2], CAN [3], FlexRay [4][5], MOST and IDB-1394 [7][8]. This has led to the concept of multiplexing, whose principal advantage is a significant reduction in the wiring cost as well as the flexibility it gives to designers; data (e.g. vehicle speed) sampled by one microcontroller becomes available to distant functions that need them with no additional sensors or links. Furthermore, while the reliability and the performance of hardware components are increasing, their cost is decreasing. On the other hand, several new functions whose development would be costly or even not feasible if using only mechanical or hydraulic technology can be realized thanks to software technology, satisfying therefore the end user requirements in terms of performance, cost, safety and comfort. In short, thanks to these technologies, the customers can buy a safe, efficient and personalised vehicle while the carmakers are able to master the differentiation of product variants and the innovation (in 2002, analysts stated that more than $80 \%$ of added value, will be obtained thanks to electronic and software-based systems [9]). A new factor, multimedia and telematics in vehicles, is emerging. This domain includes systems that support information exchanges inside a vehicle, between vehicles or between vehicle and road infrastructures. Even if these kinds of applications have little to do with the vehicle's operation itself, they grow significantly the part of software embedded in a car and require a high level of realtime quality of service and an important increase of the computation power.

The development of an embedded electronic architecture is a cooperative work involving an OEM (Original Equipment Manufacturer, or carmaker) and several Tier 1 suppliers. The result of this work is a complex distributed system, integrating several heterogeneous communication networks, and subject to stringent safety requirements.

In the near future, the development process of in-vehicle embedded systems will be leaded by the safety concerns because of the emerging ISO 26262 standards [10] that are likely to influence the certification process for embedded systems of the automotive domain in terms of dependability guarantee. Nevertheless, in a highly competitive domain like the automotive industry, the design and production costs must be kept as low as possible. In this context, it is vital for the different stakeholders to master the complexity of the design of the embedded electronic architecture. One way to reach this goal is to use a modelling language capable of representing the system at all its design steps and common to all the actors involved in the design process (such languages have been developed: AIL_Transport [11], EAST-ADL [12], EASTADL2 [13].) These languages are mainly based on a reference architecture of the embedded systems. A reference architecture defines the overall organization and the models of computation and communication used to implement computerbased systems of a certain domain. A first study was carried out within the TTA project, which focused on time-triggered architecture [14]. The proposal was intended to ease "composability" and to provide a transparent implementation of fault-tolerance mechanisms. A similar target is the first objective of the international program AUTOSAR [15][16], which gathers the majority of the stakeholders of the automotive industry in the world. One of the goals of the AUTOSAR project is to make it possible to separate software from hardware in the design process of a complex distributed technical architecture, thus allowing the portability of application software components, the flexibility for product modification, upgrade and update, the scalability of solutions within and across product lines, and the improvement of the quality and reliability of embedded systems.

In this global context of standardization, the AUTOSAR reference architecture and the ISO 26262 safety standard, two problems have to be tackled simultaneously: the integration of components (hardware but mainly software) and the guarantee that the resulting global system will not jeopardize the safety of the vehicle while ensuring a low cost for the carmakers as well for the customers, and a high level of performance and comfort. Therefore, the keywords are "composability", portability, safety assessment, real-time assessment and schedulability analysis for tasks and messages.

This Special Section on "In-Vehicle Embedded Systems" presents four papers that deal with relevant aspects pertaining to the topics highlighted above, covering a spectrum of topics, ranging from schedulability and real-time Quality of Service analysis, thanks to deterministic as well to stochastic approaches, to the composability issues when components provided by different partners are to be integrated or when the designer wants to obtain preliminary results on end-to-end response time during earlier phases of the design process.

The paper "A Synchronization Protocol for Temporal Isolation of Software Components in Vehicular Systems", by Thomas Nolte, Insik Shin, Mikael Sjodin and Moris Behnam, 
deals with the temporal interoperability issue when integrating functions that are developed independently. The problem is there to guarantee a predictable behavior of the whole system. The proposed technique is based on the temporal isolation of the different functions and is specifically well suited to the AUTOSAR context. A schedulability analysis completes the proposed mechanisms.

It can be difficult and even not possible to compute a tight upper bound for end-to-end response time of a complex function distributed onto several ECUs communicating through a network. Therefore a probabilistic approach [17] is a good way to obtain valuable information in the early stage of the architecture design. How to evaluate the probability distribution of the end-to-end latencies in a CAN-based architecture is the topic of the paper "Stochastic Analysis of Distributed Real-time Automotive Systems" proposed by Haibo Zeng, Marco Di Natale, Paulo Giusto and Alberto Sangiovanni-Vincentelli.

Several economic and technical reasons justify the introduction of multicore nodes in a vehicle. First a large amount of functions require an always increasing computation power (complex control laws or multimedia streams handling). Then, each core can be dedicated to specific functions such as, for example, the AUTOSAR communication stack or specific sensors handling functions. Finally, these new architectures can support the deployment of safety mechanisms (redundancy of components, active / passive control of software components, etc.). Safety and realtime assessment as well as deployment optimization are two problems that have to be taken into account in this new context [18]. The paper "Response Time Analysis in Multicore ECUs with Shared Resources" from Simon Schliecker, Mircea Negrean and Rolf Ernst shows how to ensure the predictability of real-time requirements. In particular it develops an analytical approach in order to determine the worst-case response time for tasks allocated on a multicore ECU with shared resources.

The paper "Traffic Shaping for Resource-Efficient InVehicle Communication" from M. Rahmani, K. Tappayuthpijarn, B. Krebs, R. Bogenberger and E. Steinbach investigates the impact of the introduction of IP-based network architecture in in-vehicle embedded architecture. More precisely, they propose to deploy multimedia applications onto a full-duplex switched Ethernet. For such application, the challenge is to guarantee the real-time Quality of Service for the transmission of audio and video stream. The authors propose a novel traffic shaping policy suited to these applications, evaluate its performance and detail how to implement it.

I would like to thank all of the authors, for their contributions and their co-operation in promptly replying to the reviewers' comments, the reviewers, for their careful reviews which contributed in a significant way to the quality level of the papers that were published and, finally, the editors of these Transactions, for their guidance in preparing and finalizing this special section, from the very first steps until its publication.

FRANÇOISE SIMONOT-LION, Guest Editor

University of Nancy, LORIA (UMR 7503)

54506 Vandœuvre-lès-Nancy, France (imonot@loria.fr)

\section{REFERENCES}

[1] www.sae.org.

[2] LIN Consortium, LIN Specification Package, revision 2.1, November 2006, Available at http://www.lin-subbus.org/

[3] ISO/DIS 11898, Road Vehicles - Interchange of Digital Information Controller Area Network (CAN) for High Speed Communication, 1992..

[4] FlexRay Consortium, FlexRay Communications System - Protocol Specification, Version 2.1, 2005. Available at http://www.flexray.com..

[5] B. Schätz, C. Kühnel, Gonschorek, The FlexRay Protocol, in Automotive Embedded Systems Handbook (Ed. N. Navet and F. Simonot-Lion), CRC Press/Taylor and Francis, December 2008.

[6] http://mostnet.de/.

[7] www.idbforum.org

[8] www.1394ta.org

[9] G. Leen, D. Heffernan, Expanding Automotive Electronic Systems, Computer, Vol.35, N¹, pp. 88-93, IEEE Computer Society, 2002.

[10] H. Schubotz, Experience with ISO WD 26262 in automotive safety projects, SAE World Congress \& Exhibition, Detroit, MI., April 2008.

[11] J.-P. Elloy, F. Simonot-Lion, An architecture description language for in-vehicle embedded system development, 15th IFAC World Congress, Barcelona, Spain, 2002.

[12] U. Freund, O. Gurrieri, J. Küster, H. Lönn, J. Migge, M.-O. Reiser, et al., An architecture description language for developing automotive ECUsoftware, International Conference On Systems Engineering (INCOSE 2004), Toulouse, France, 2004.

[13] P. Cuenot, P. Frey, R. Johansson, H. Lönn, M-O Reiser, D. Servat, R. Tavakoli Koligari, D.J. Chen. Developing automotive products using the EAST-ADL2, and Autosar compliant architecture description language. Embedded Real-Time Software Conference, Toulouse, France, 2008.

[14] C. Scheidler, G. Heiner, R. Sasse, E. Fuchs, H. Kopetz, C. Temple, Time-Triggered Architecture (TTA), Multimedia, Embedded Systems and Electronic Commerce Conference, Florence, Italy, 1997.

[15] AUTOSAR GbR, Technical Overview, V3.0. Available at: http://www.autosar.org, 2008.

[16] S. Voget, M. Golm, B. Sanchez, F. Stappert, Application of the AUTOSAR standard. In N. Navet, F. Simonot-Lion (Eds.). Automotive Embedded Systems Handbook, Taylor\&Francis, 2008.

[17] J. L. Díaz, D. F. García, K. Kim, C.-G. Lee, L. Lo Bello, J. M. López, S. L. Min, O. Mirabella, Stochastic Analysis of Periodic Real-Time Systems, 23rd IEEE Real-Time Systems Symposium (RTSS'02), 2002.

[18] H. Kopetz, R. Obermaisser, C. El Salloum, B. Huber, Automotive Software Development for a Multi-Core System-on-a-Chip, 4th International Workshop on Software Engineering for Automotive System (SEAS '07), Minneapolis, May 2007.

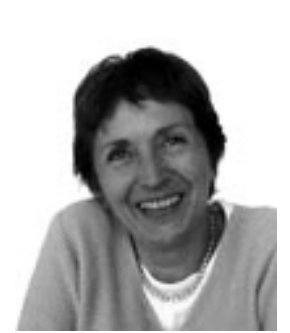

Françoise Simonot-Lion is Professor of Computer Science at Nancy Université (France). Since 1997, she is the scientific leader of the Real Time and InterOperability (TRIO) research team, an INRIA project at LORIA laboratory in Nancy (France). Its main research topics are on the one hand, modeling and verification techniques for the design of optimized real time distributed applications under safety constraints, and on the other hand, specification of embedded services ensuring a real time Quality of Service (scheduling of tasks and messages, real time middleware, frame packing).

She served as Program Co-Chairman for the 2008 edition of the IEEE Workshop on Factory Communication Systems. She co-authored more than 70 technical paper in the area of real-time systems modeling and analysis. She is co-chair of the subcommittee "Automotive Electronic and Embedded Systems” of the IEEE Industrial Electronic Society (IES) - TCFA. 